\title{
Diagnosis and management of urinary tract infection in adults
}

\author{
Martin E Wilkie, Michael K Almond, Frank P Marsh \\ Although most urinary tract infections in adults are \\ uncomplicated and respond rapidly to empirical anti- \\ microbial therapy, they represent a considerable health \\ problem, particularly among women, in whom the \\ annual general practice consultation rate for cystitis \\ and urinary infection has been estimated as $62 \cdot 5 / 1000$. \\ Occasionally, urinary tract infections cause serious and \\ permanent renal damage in patients with underlying \\ abnormalities of the renal tract, pregnancy, or diabetes \\ mellitus. ${ }^{2}$ In young men who have not had instru- \\ mentation of the genitourinary tract urinary tract \\ infection is rare, except in sexually active homosexuals,

\section{Indications for an intravenous pyelogram} \\ - First infections in men \\ - Recurrent infections in women \\ - Overt haematuria \\ - Persistent microscopic haematuria \\ - Acute pyelonephritis in systemic disease \\ - The identification of an unusual organism \\ - Fever not settling within 48 hours of treatment
} but it is more common after the age of 50 owing to prostatic disease. In institutionalised elderly patients the prevalence of asymptomatic bacteriuria approaches $20-50 \%$ in women and $5-20 \%$ in men. ${ }^{34}$ Urinary tract infections in childhood may cause longlasting and progressive renal damage, which may result in end stage renal failure in young adults.

\section{Diagnosis}

Urinary frequency, dysuria, and suprapubic discomfort are the characteristic symptoms of cystitis, often causing considerable distress with sleep disturbance, time off work, and inability to participate in activities or travel. The differential diagnosis of these symptoms includes the urethral syndrome,' sexually transmitted disease, and vaginitis. ${ }^{\circ}$

In general, the patient with acute pyelonephritis is systemically unwell with rigors, fever, back and loin pain with tenderness in the costovertebral angle, colicky abdominal pain, nausea, vomiting, and leucocytosis. Blood and urine cultures usually have positive results, and Gram negative shock may occur, particularly when there is complicating obstructive uropathy due to papillary necrosis.

Department of Nephrology, The Royal London Trust, London E1 1BB

Martin E Wilkie, registrar Michael K Almond, senior registrar

Frank P Marsh, consultant nephrologist

Correspondence to: Martin E Wilkie, Northern General Hospital NHS Trust, Herries Road, Sheffield S5 7AU

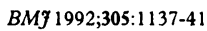

\section{Bacteriology}

Classically, the diagnosis of urinary tract infection is dependent on culturing a microorganism from the urine. This simple statement, however, belies the complexity of the problem. How should the urine be collected, what quantity of organisms in the urine is regarded as being clinically important, how does one approach the problem of sterile urine but troublesome symptoms, and is bacteriological diagnosis really necessary?

On the basis of the work of Kass, $10^{5}$ colony forming units per millilitre in a midstream sample of urine became regarded as "significant bacteriuria"the threshold for distinguishing urinary tract infection from bacterial contamination. Contamination becomes more likely if there is a mixed growth of bacteria, and less likely if the same organism is cultured on more than one occasion or if the sample is from a male patient. In certain circumstances a lower threshold should be considered as significant-for example, where the organism is more difficult to grow (Staphylo- coccus saprophyticus, chlamydia, mycobacterium), or if the specimen has been collected from a urinary catheter. The presence of any organisms in a sample taken by direct bladder aspiration indicates an infection. Specimens need to be transported to the laboratory for culture without delay, and if stored for a few hours should be kept at $4^{\circ} \mathrm{C}$ to prevent bacterial proliferation artificially increasing the count. Automated culture systems are available, requiring 5-13 hours to detect significant bacteriuria, and some of these methods will allow antibacterial susceptibilities to be determined. These methods have low sensitivity for detecting urinary tract infections with low colony count and are not applicable to the hospital setting, where the responsible organisms are often slow growing. ${ }^{7}$ Direct microscopy is a readily available but uncertain method of determining bacteriuria.

Pyuria is supportive evidence of the presence of a clinically important urinary tract infection, but pus can be present in "sterile" urine, where it may indicate a fastidious organism such as mycobacterium or recent treatment with antibiotics. Pyuria can be detected by the leucocyte esterase test, which has been incorporated onto a diagnostic stick; when combined with a positive nitrate test this test has $87 \%$ sensitivity and $67 \%$ specificity in detecting urinary tract infections due to enterobacteria. ${ }^{8}$ Microscopic haematuria is present in $50 \%$ of urinary tract infections. ${ }^{6}$ The presence of albumin on a dip stick is not a good indicator of urinary tract infection.

Host susceptibility to a range of virulence factors expressed by the infecting organism, including the avidity of microbacterial adherence to the urothelium, may explain why some women with urinary tract infection are predisposed to recurrence and pyelonephritis while others are not. ${ }^{9}$ Nearly $90 \%$ of community acquired urinary tract infections in women are due to Escherichia coli; other Gram negative bacteria such as Proteus mirabilis and klebsiella species are occasionally responsible (fig 1). Saprophytic staphylococci account for $10 \%$ of urinary tract infections in sexually active women; enterococci are occasionally isolated. Community acquired organisms are generally susceptible to commonly used antimicrobial agents. In contrast, $E$ coli is responsible for only half of 
the hospital acquired infections with Pseudomonas aeruginosa, proteus species, klebsiella species, serratia, enterobacter, enterococci, staphylococci, yeasts, and many other organisms accounting for the remainder (fig 1). Furthermore, multiple antibiotic resistance is commonly seen in these infections. Gram positive organisms were cultured in $39 \%$ of elderly men with urinary tract infections whereas enterobacteria (escherichia, klebsiella, proteus) accounted for $93 \%$ of isolates in elderly women. ${ }^{3}$ Anaerobic bacteria are rare and are associated with obstruction, often due to malignant disease. ${ }^{8}$

Candiduria occurs in diabetic patients, in those who have been taking antibiotics, and after long term catheterisation. In immunosuppressed individuals it may be the first indicator of systemic candidiasis. ${ }^{8}$

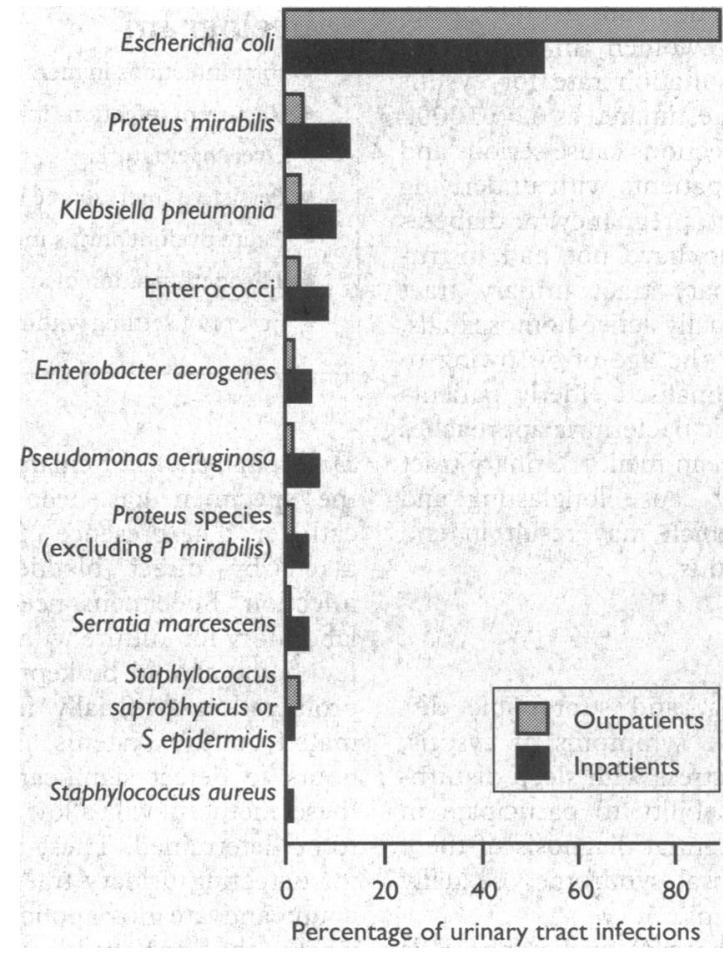

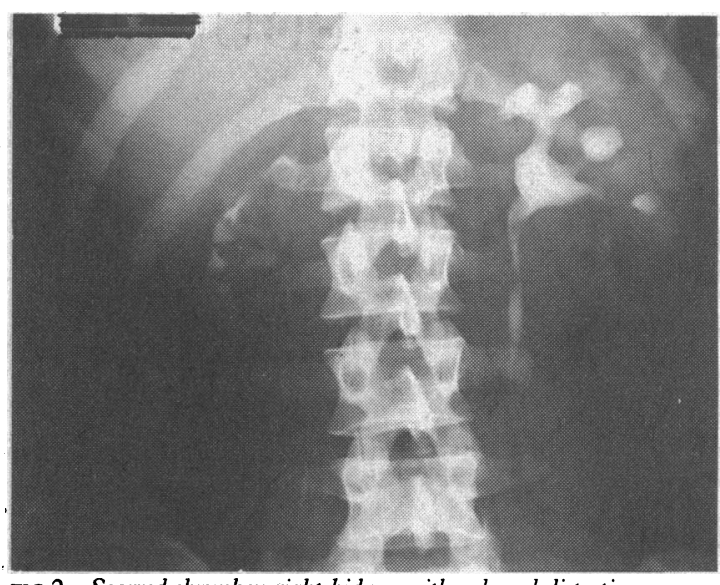

FIG 2-Scarred shrunken right kidney with calyceal distortion seen on intravenous pyelogram; typical appearances of chronic pyelonephritis

\section{Radiology}

Radiological abnormalities are rarely found in premenopausal women with no features in their history to suggest an underlying cause of the infection. ${ }^{10} \mathrm{~A}$ plain abdominal film may show calculi or renal calcification due to tuberculosis or renal tubular acidosis. An intravenous pyelogram (fig 2 ) is indicated for recurrent infections, continued symptoms despite treatment, overt haematuria, persistent microscopic haematuria, first infections in men, acute pyelonephritis in patients with underlying systemic disease which increases their risk of complications, ${ }^{11}$ or where the infecting organism is unusual or fever does not settle within 48 hours of starting antibiotic treatment. ${ }^{12}$ Ultrasonography is particularly useful in the detection and percutaneous drainage of hydronephrosis (fig 3) and renal abscesses, or where an intravenous pyelogram is not possible due to renal functional impairment. Ultrasonography also has the advantage of being able to detect pelvic pathology. The investigation of vesicoureteric reflux requires a voiding cystourethrogram ${ }^{13}$ or an indirect radionuclide cystogram using myelin associated glycoprotein labelled with technetium-99m ( ${ }^{99 m}$ Tc MAG3) which has the advantages of reduced radiation dosage and avoiding bladder catheterisation. ${ }^{14}$ The optimal radiological investigations to detect renal scars include both an intravenous urethrogram and a ${ }^{99 \mathrm{~m}} \mathrm{Tc}$ dimercaptosuccinic acid (DMSA) renal scan (fig 4).

\section{Management}

A careful history should determine the nature of the infection and guide the doctor in deciding the duration and type of treatment and the requirement for further investigations. Is this the first infection, a relapse (reappearance of the same serotype), or a reinfection (a different infecting organism from the faecal reservoir); is it associated with renal tract instrumentation or prostatism; or is there a history to suggest renal pathology (urinary tract infection in childhood, hypertension in pregnancy)?

\section{ANTIMICROBIAL AGENTS-DOSE AND DURATION}

Antimicrobial agents can cause vulvovaginal candidiasis, hypersensitivity reactions, rashes, and gastrointestinal disturbances. Limiting treatment to a minimum reduces adverse effects and cost while increasing compliance. Women with bacterial cystitis may be treated just as effectively with a short course, or even a single high dose, of an appropriate antimicrobial agent as with a conventional 7-14 day course of treating infections due to the two most common organisms to infect women, $E$ coli and $S$ saprophyticus, but infections with $P$ mirabilis and Streptococcus faecalis have a disappointing cure rate $(46 \%)$. The major disadvantage with single dose therapy for urinary tract infection is that it is inadequate for eradicating uropathogens from faecal and vaginal reservoirs; hence recurrence is more common. Thus short course therapy of 3-5 days may be better for recurrent urinary tract infection. Women with a history of multiple recurrences in the previous year should receive a six week regimen. Short course therapy should never be

\section{Short course antimicrobial chemotherapy}

- Highly effective in treating the two most common organisms to infect women-Escherichia coli and

\section{Should never be used for}

- Men

- Pyelonephritis

- Infections lasting over one week

- Anatomical or functional abnormalities

- Immunosuppressed patients

- Infections associated with catheters

- Pregnant women treatment. ${ }^{15}$ Single dose therapy is highly effective for Staphylococcus saprophyticus

- Reinfections (where resistance is probable) 
used for patients who have a high probability of deep tissue infection-men; patients with pyelonephritis, symptoms lasting more than seven days, anatomical or functional abnormalities of the urinary tract, immunosuppression, or indwelling catheters; and those with a high probability of having resistant bacteria. ${ }^{8}$ Indeed, the failure of a single dose treatment defines a subgroup of women with renal infection who require further urinary tract investigation, more intensive treatment, and closer supervision. ${ }^{16}$

Amoxycillin has fallen from favour as a treatment of urinary tract infection because of the development of resistant strains in the faecal flora, resulting in recurrent infection, but the addition of a $\beta$ lactamase inhibitor (augmentin) is highly effective. Trimethoprim is active against most common urinary pathogens and is particularly suitable for low dose suppression as it achieves high concentrations in the vagina, ${ }^{17}$ although recurrence due to resistant enterobacteria has been increasingly reported. Co-trimoxazole has more
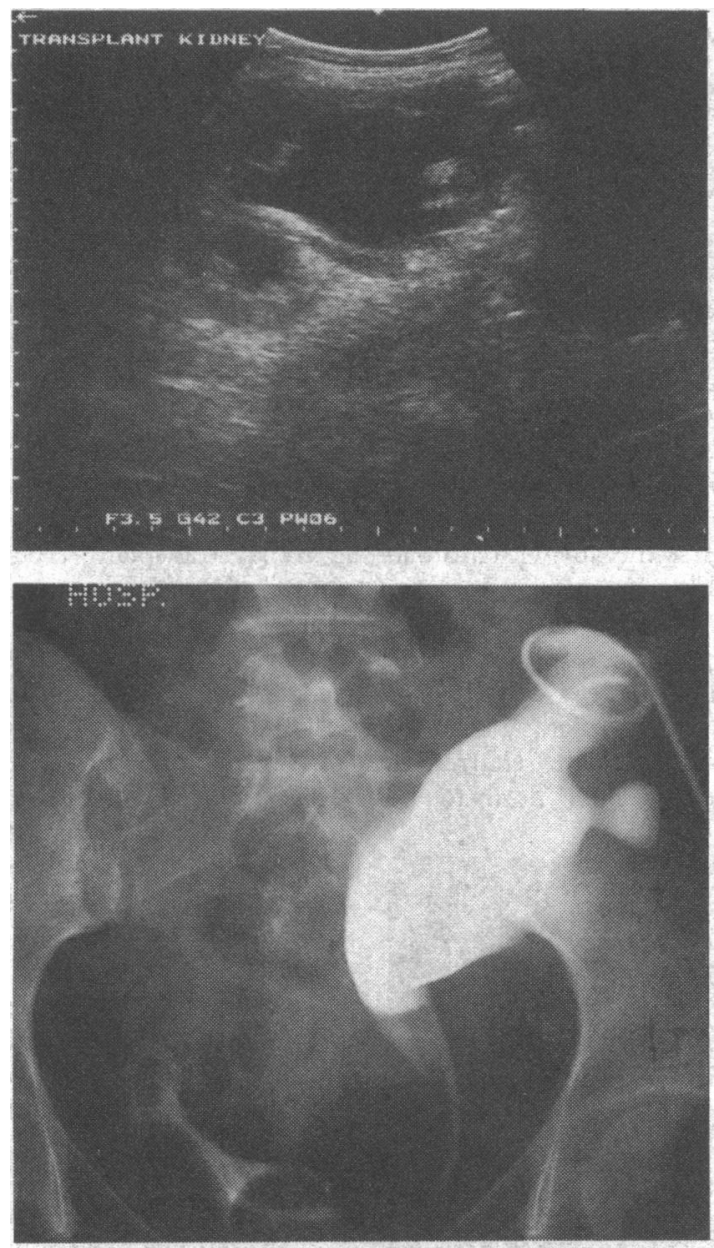

FIG 3-(a) Ultrasound scan of grossly hydronephrotic transplanted kidney; (b) antegrade nephrostogram confirming the hydronephrosis; (c) normal ultrasound appearances of transplanted kidney

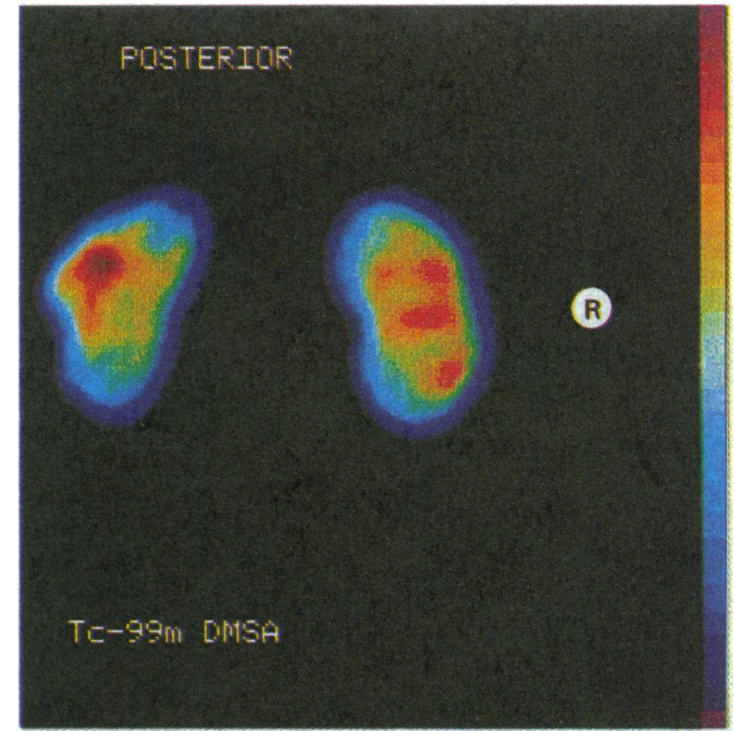

FIG 4 -99w $T c$ DMSA scan showing scarring of left kidney

side effects and little advantage in the treatment of urinary tract infections. ${ }^{18}$ The oral cephalosporins cause less resistance among bowel flora than do the penicillins and are excreted in active form in high concentrations in the urine. ${ }^{17}$

The new quinolones, such as norfloxacin and ciprofloxacin, have a broad spectrum of activity and are effective against Gram positive and Gram negative bacteria, including pseudomonas species. Quinolones are not recommended in pregnancy, and should be used with caution in epilepsy. Quinolones increase the plasma concentration of theophyllines. Simultaneous treatment with antacids, calcium, or iron reduces intestinal absorption of quinolones by half. Nitrofurantoin is concentrated in the urine, and does not promote resistance of organisms in the faecal flora; thus it is ideally suited for prophylaxis in patients with unimpaired renal function, but it should not be used where there is renal impairment.

LOWER URINARY TRACT INFECTION

Transient episodes of uncomplicated bacteriuria are common in women, particularly after sexual intercourse, and resolve spontaneously in 24-72 hours, except in pregnancy. For patients with symptomatic cystitis, antimicrobial treatment in addition to routine advice regarding fluids and voiding practices will lead to an earlier eradication of the infection and resolution of symptoms. In general practice, acute dysuria in women can be managed with a short course of an antibiotic in those with severe symptoms, reserving urine cultures and further investigation for those women who have recurring attacks. ${ }^{19}$

\section{ACUTE PYELONEPHRITIS}

Acute pyelonephritis should be treated promptly and vigorously and may require parenteral antibiotics in a hospital setting. Women with acute pyelonephritis who are able to take fluids orally and are not pregnant or diabetic and have no other complicating factors may be treated at home. ${ }^{12}$ If the antibacterial sensitivities of the infecting organism are known, full doses of the least toxic agent should be prescribed. Broad spectrum regimens usually have to be prescribed empirically until the results of blood and urine cultures become available. Second or third generation cephalosporins, ciprofloxacin, co-trimoxazole, trimethoprim, or broad spectrum penicillins such as ticarcillin with clavulanic acid $^{17}$ have been recommended. Aminoglycosides are cheap and effective against most Gram negative organisms, but plasma levels must be carefully monitored to avoid toxicity. Antibacterial sensitivities are 
usually available within 48 hours, and the toxicity of aminoglycosides over this period is negligible if there is no underlying renal impairment. Parenteral administration can usually be changed to an appropriate oral antibiotic 48 hours after the fever has settled and treatment should be continued for a further two weeks. It is important to exclude underlying predisposing disorders such as diabetes, renal tract abnormalities, or neurogenic bladder (due to multiple sclerosis, for example).

\section{INFECTIONS IN PREGNANCY}

Screening for bacteriuria is indicated in pregnancy. More than a quarter of patients with bacteriuria later develop symptomatic urinary tract infection or acute pyelonephritis if left untreated. Continuing bacteriuria is associated with premature delivery and increased perinatal mortality. Although single dose therapy is attractive for the treatment of bacteriuria in pregnancy as it minimises exposure of the fetus to potentially toxic agents, it has not yet been proved to be adequate. The current recommendation is for a 7-14 day course of a relatively non-toxic drug such as a sulphonamide, ampicillin, amoxycillin, or cephalexin..$^{20}$ Sulphonamides should be avoided in late pregnancy because of the risk of kernicterus. Treatment should be followed by a repeat urine culture to confirm clearing of the organism..$^{21}$

\section{The current}

recommendation is for a 7-14 day course of a choice of

- Ampicillin

- Amoxycillin

- Cephalexin

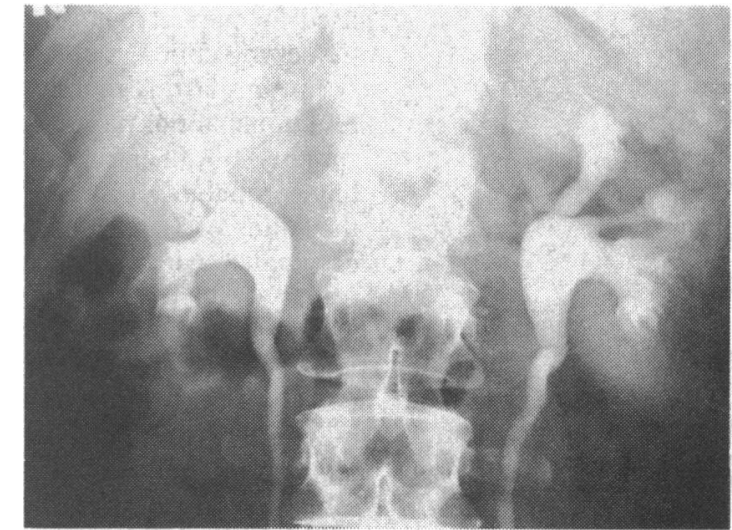

FIG 6-Bilateral papillary necrosis with contrast extending into rena papillae, indicating fissuring

replaced by lactobacilli. ${ }^{3}$ Prostatitis is not uncommon in elderly men and requires at least four weeks' treatment with co-trimoxazole, ciprofloxacin, ${ }^{3}$ or erythromycin to penetrate tissues adequately.

\section{PREDISPOSING CONDITIONS}

Abnormalities of the urinary tract that disturb the normal hydrokinetic defence mechanisms predispose both sexes to urinary tract infections (fig 5). Infection in the presence of obstruction, calculus, or abnormalities of bladder emptying can result in severe impairment of renal function. The prevalence of bacteriuria is increased in diabetic patients, ${ }^{23}$ who have a greater risk of developing complications such as pyelonephritis, papillary necrosis (fig 6), septicaemia, and renal abscesses. Raised urinary glucose concentrations, defects in leucocyte function, obesity, vulvovaginitis, and bladder neuropathy may be causes of urinary tract infections.

Renal transplant recipients have anatomical and immunological reasons for developing urinary tract infections, which occur in $30-50 \%$ of these patients. ${ }^{24-20}$ Vesicoureteric reflux of infected urine into the transplanted kidney often causes pyelonephritis ${ }^{24} 27$ with bacteraemia and can trigger acute rejection. Where possible, urine cultures should be taken before operation and continued regularly in the immediate postoperative period, and bacteriuria should be treated. It is important that the duration of urinary catheterisation is kept as short as possible.

\section{URINARY CATHETERS}

Indwelling catheters are implicated in $40-75 \%$ of all hospital acquired urinary tract infections-a leading cause of Gram negative bacteraemia. Careful catheter care and the use of a catheter team reduces infection rate, but local disinfection regimens have not been helpful. For catheters in place for up to 14 days prophylactic antimicrobial agents reduce the risk of bacteriuria fivefold, ${ }^{28}$ but when catheters are used longer than this the frequency with which resistant organisms appear has led to the recommendation to treat only symptomatic bacterial infections. ${ }^{29}$ Management of candidal colonisation of the bladder includes removal of the catheter, stopping antibiotics, and irrigation with amphotericin. ${ }^{30}$ Intermittent self catheterisation using a clean technique has benefits for a wide range of patients with atonic or neuropathic bladders, ${ }^{31}$ including paraplegia patients in spinal units, and eliminates cross infection. ${ }^{32}$

\section{"PROPHYLAXIS"}

Continuous low dose suppression is effective at reducing recurrence rates for urinary tract infection by $95 \%$ among sexually active women. Co-trimoxazole, trimethoprim alone, nitrofurantoin, and norfloxacin in low dose at night are well documented to be efficacious 


\section{Antimicrobial chemoprophylaxis}

\section{Recommended}

- For women with recurrent infections

- After acute pyelonephritis in pregnancy

- Before instrumentation of the urinary tract for short term urinary catheterisation

- Before extracorporeal shock wave lithotripsy

with a low incidence of side effects in long term therapy. ${ }^{8}$ Suppression is recommended for women who experience troublesome recurrence of infections and is generally given for six or 12 months but has been effective for two to five years with no increase in adverse effects. ${ }^{33}$ Infections occurring during suppression are usually resistant to the antimicrobial agents being taken, and an alternative appropriate drug should be selected on the basis of the bacterial sensitivities. An alternative to long term continuous suppression is postcoital treatment, which should be combined with postcoital voiding in women who identify this as a precipitating factor for infections. Early self treatment with a broad spectrum antibiotic for symptoms of urinary tract infection is also effective in women with recurrent infections. ${ }^{34}$ Low dose suppression has also been recommended following acute pyelonephritis in pregnancy as recurrence rates of $10-25 \%$ have been reported. ${ }^{35}$

Treatment with antibiotics before instrumentation of the urinary tract prevents bacteraemia and Gram negative septicaemia. Antibiotics should also be given before extracorporeal shock wave lithotripsy to prevent infection by viable organisms retained in stone fragments. ${ }^{36}$

1 Royal College of General Practitioners, Office of Population Censuses and Surveys, Department of Health and Social Security. Morbidity statistics from general practice. Third national study 1981-82. London: HMSO, 1986:159.

2 Hooton TM, Stamm WE. Management of acute uncomplicated UTI in adults. Med Clin North Am 1991;75:339-57.

3 Baldassarre JS, Kaye D. Special problems of urinary tract infection in the elderly. Med Clin North Am 1991;75:375-90.

4 Schaeffer AJ. Urinary tract infections in the elderly. Eur Urol 1991;19(suppl

5 Brumfitt W, Hamilton-Miller JMT, Gillespie WA. The mysterious "urethral syndrome." $B M \mathcal{F}$ 1991;303:1-2.

6 Stamm WE. Protocol for the diagnosis of urinary tract infection: reconsidering the criterion for significant bacteriuria. Urology 1988;32(suppl 2):6-12
7 Pappas PG. Laboratory in the diagnosis and management of urinary tract infections. Med Clin North Am 1991;75:213-25.

8 Rubin RH, Tolkoff-Rubin NE, Cotran RS. Urinary tract infection, pyelonephritis and reflux nephropathy. In: Brenner BM, Rector FC, eds. The Kidney Vol II. Philadelphia: W B Saunders, 1991:1369-429.

9 Johnson JJ. Virulence factors in Escherichia coli urinary tract infection. Clin Microbiol Rev 1991;4:80-128.

$10 \mathrm{McNicholas}$ MMJ, Griffin JF, Cantwell DF. Ultrasound of the pelvis and renal tract combined with a plain film of abdomen in young women with urinary tract infection: can it replace intravenous urography? A prospective urinary tract infection: can it replace

11 Merenich WM, Popky GL. Radiology of renal infection. Med Clin North Am 1991;75:425-69.

12 Patton JP, Nash DB, Abrutyn E. Urinary tract infection: economic considerations. Med Clin North Am 1991;75:495-513.

13 Arant BS. Vesicoureteric reflux and renal injury. Am $\mathcal{Y}$ Kidney Dis 1991;17: 491-511.

14 Gordon I. Urinary tract infection in paediatrics: the role of diagnostic imaging. Br F Radiol 1990;63:507-11.

15 Bailey RR. Review of published studies on single dose therapy of urinary tract infections. Infection 1990;18(suppl 2):53-6.

16 Ronald AR, Conway B, Zhanel GC. The value of single-dose therapy to diagnose the site of urinary infection. Chemotherapy 1990;36(suppl 1):2-9.

17 Iravani $\mathrm{A}$. Advances in the understanding and treatment of urinary tract infections in young women. Urology 1991;37:503-11.

18 Co-trimoxazole, or just trimethoprim? Drug Ther Bull 1986;24:17-9.

19 Brooks D. The management of suspected urinary tract infection in general practice. Br $\mathcal{F}$ Gen Pract 1990;40:399-401.

20 Cephalosporins - what's changed? Drug Ther Bull 1986;24:101-4.

21 Andriole VT, Patterson TF. Epidemiology, natural history, and management of urinary tract infections in pregnancy. Med Clin North Am 1991;75:359-73.

22 Rocco F, Franchini V. Antimicrobial therapy for treatment of UTI in the elderly. Eur Urol 1991;19(suppl 1):7-15.

23 Keane EM, Boyko EJ, Reller LB, Hamman RF. Prevalence of asymptomatic bacteriuria in subjects with NIDDM in San Luis Valley of Colorado. Diabetes Care 1989;11:708-12.

24 Dunn SP, Vinocur CD, Hanevold C, Wagner CW, Weintraub WH. Pyelonephritis following paediatric renal transplant: increased incidence with vesicoureteral reflux. F Pediatr Surg 1987;22:1095-9.

25 Hansen BL, Rohr N, Svendsen V, Olsen H, Birkeland SA. Bacterial urinary tract infection in cyclosporine-A immunosuppressed renal transplant recipients. Scand $\mathcal{F}$ Infect Dis 1988;20:425-7.

26 Cohen J, Rees AJ, Williams G. A prospective randomised controlled trial of perioperative antibiotic prophylaxis in renal transplantation. $\mathcal{Y}$ Hosp Infect 1988;11:357-63.

27 Hanevold CD, Kaiser BA, Palmer J, Polinsky MS, Baluarte HJ. Vesicoureteral reflux and urinary tract infections in renal transplant recipients Am F Dis Child 1987;141:982-4.

28 Hustinx WNM, Minties-de Groot AJ, Verkooyen RP, Verbrugh HA. Impact of concurrent antimicrobial therapy on catheter-associated urinary tract infection. I Hosp Infect 1991;18:45-56.

29 Sanderson PJ. Prophylaxis for catheter related urinary tract infection. f Hosp Infect 1991;18:1-3.

$30 \mathrm{Hsu}$ CCS, Ukleja B. Clearance of candida colonizing the urinary bladder by a two-day amphotericin B irrigation. Infection 1990;18:280-2.

31 Underused: self intermittent catheterisation. Drug Ther Bull 1991;29:37-9.

32 Wyndaele JJ, De Taeye N. Early intermittent self-catheterisation after spinal cord injury. Paraplegia 1990;28:76-80.

33 Stamm WE, McKevitt M, Roberts PL, White NJ. Natural history of recurrent urinary tract infections in women. Rev Infect Dis 1991;13:77-84

34 Nicolle LE. The optimal management of lower urinary tract infections. Infection 1990;18(suppl 2):50-2.

35 Sandberg T, Brorson JE. Efficacy of long-term antimicrobial prophylaxis after acute pyelonephritis in pregnancy. Scand $\mathcal{I}$ Infect Dis 1991;23:221-3.

36 Michaels EK, Fowler JE, Mariano M. Bacteriuria following extracorporeal shockwave lithotripsy of infection stones. $\mathcal{F}$ Urol 1988;140:254-6.

\title{
London After Tomlinson
}

\section{Care in the capital: what needs to be done}

\author{
David Metcalfe
}

This is the second article in our series looking at the issues highlighted by the Tomlinson report into London's health care and medical research and education

\section{Department of General Practice, University of Manchester, Manchester M14 5NP David Metcalfe, professor}

BMO 1992;305:1141-4

\section{Summary}

One of the aims of the Tomlinson report is to shift more care from the secondary to the primary sector in London. But the primary sector is already underresourced and overloaded. The capital has a heterogeneous population which often makes inappropriate demands on general practitioners. Many premises are inadequate and there are insuffcient support staff. David Metcalfe emphasises that London is special and that the shift will not become a reality unless these problems are tackled. He suggests the establishment of different models of practice centres which could treat some of the patients who now go to accident and emergency departments. Some would be the night emergency service base, some would have primary care beds, and each would have a different mix of specialist support.

Primary care has been used to describe a variety of activities, but most meanings stem from the idea of first contact care, with referrals to secondary care by more specialised personnel with better resources. They may in turn refer onwards for tertiary care. In Britain the nub of the NHS has been open access to general practitioners and to those who work alongside them to provide medical care outside the hospital, such as district nurses and health visitors.

These professionals do not only provide first contact care; their training equips them to look after people with chronic diseases, to practise preventive medicine, and to care for the dying and support their families. 